\title{
Accrete, Accrete, Accrete... Bang! (and repeat): The Remarkable Recurrent Novae
}

\author{
Matthew J. Darnley* \\ Astrophysics Research Institute, Liverpool John Moores University, IC2 Liverpool Science Park, \\ 146 Brownlow Hill, Liverpool, L3 5RF, UK \\ E-mail: M.J.Darnley@l jmu.ac.uk
}

\begin{abstract}
All novae recur, but only a handful have been observed in eruption more than once. These systems, the recurrent novae ( $\mathrm{RNe}$ ), are among the most extreme examples of novae. RNe have long been thought of as potential type Ia supernova progenitors, and their claim to this 'accolade' has recently been strengthened. In this short review RNe will be presented within the framework of the maximum magnitude - rate of decline (MMRD) phase-space. Recent work integrating Heflashes into nova models, and the subsequent growth of the white dwarf, will be explored. This review also presents an overview of the Galactic and extragalactic populations of RNe, including the newly identified 'rapid recurrent nova' subset - those with recurrence periods of ten years, or less. The most exciting nova system yet discovered - M31N 2008-12a, with its annual eruptions and vast nova super-remnant, is introduced. Throughout, open questions regarding $\mathrm{RNe}$, and some of the expected challenges and opportunities that the near future will bring are addressed.
\end{abstract}

The Golden Age of Cataclysmic Variables and Related Objects V (GOLDEN2019)

2-7 September 2019

Palermo, Italy

${ }^{*}$ Speaker. 


\section{Introduction}

Nova eruptions rank among the most luminous stellar explosions yet discovered. They are surpassed in brightness by only $\gamma$-rays bursts and the full gamut of supernovae ( $\mathrm{SNe}$; see, for e.g., [1]). Yet novae are substantially more numerous, with $50_{-23}^{+31}$ eruptions every year in the Milky Way [2], $65_{-15}^{+16} \mathrm{yr}^{-1}$ in the Andromeda Galaxy (M 31) [3], and as many as 300 annually in M 87 [4, 5]; compared to $\sim 1 \mathrm{SN}$ per century per host. Like all explosive transients, novae enrich the ISM; in this case through the production of ${ }^{7} \mathrm{Li},{ }^{13} \mathrm{C},{ }^{15} \mathrm{~N}$, and ${ }^{17} \mathrm{O}$ (e.g., [6, 7, 8, 9]).

Novae are binary systems. At their heart is a white dwarf (WD) that accretes material from a nearby companion star - the donor. For main sequence or sub-giant donors, mass-loss occurs via Roche-lobe overflow, and the system is considered a cataclysmic variable (CV). If the donor is a red giant, mass-loss is generally driven by that star's stellar winds, and the system is classed as a symbiotic variable [10]. In a typical system, material lost from the donor accumulates in an accretion disk around the WD before it is transported to the surface of the later. However, in the presence of a strong WD magnetic field accretion can be partially (intermediate polar) or entirely (polar) channeled via that magnetic field. The reader is referred to [11,12] for compendia of recent detailed and comprehensive reviews of the nova phenomenon.

Once a critical quantity of material has accreted on to the WD surface, the conditions for nuclear burning are met, which ultimately leads to a thermonuclear runaway under the degenerate conditions of the WD $[9,13,14]$. Once the temperature has increased sufficiently to lift that degeneracy, the rapid expansion of the accreted envelope leads to the ejection of a portion of the envelope. The system then enters a period of quasi-stable nuclear burning on the WD surface until the available fuel source is exhausted [15].

The gross observable features of novae are powered by the nuclear burning. Until recently (see [16]), it was understood that the ejected and expanding envelope acts solely as a photon convertor. The rapid rise in optical luminosity is driven by the expansion of an optically thick envelope. The slower optical decline from peak is linked to the decreasing optical depth of the then optically thin envelope. Subtleties of the observed features relate to factors such as shocks, ejecta photoionisation and recombination, geometry, inclination, and molecule/dust formation. See [17] for an overview.

Novae are inherently recurrent in nature. A nova eruption does not irreversibly impact the system as a whole - although the accretion disk is understood to be disrupted or destroyed in most cases (see, for e.g., [18] versus [19]). Post-eruption, the disk will recover or reform [20], accretion will resume [21], and a second eruption will occur once the WD has again accreted a critical mass of material. Theoretical work suggests that recurrence periods range from $50 \mathrm{~d}<P_{\text {rec }} \lesssim 10^{6} \mathrm{yrs}$ $[22,23,24]$. A value of a century arbitrarily delimitates the nova population into classical novae $\left(\mathrm{CNe} ; P_{\mathrm{rec}}>100 \mathrm{yrs}\right)$ and recurrent novae $\left(\mathrm{RNe} ; P_{\mathrm{rec}}<100 \mathrm{yrs}\right)$.

This article provides a summary of an invited review talk on the topic of 'Recurrent Novae', presented at The Golden Age of Cataclysmic Variables and Related Objects V meeting in Palermo, Italy (Sep. 2019) ${ }^{1}$. In Section $2 \mathrm{RNe}$ are explored in the context of the 'MMRD' relationship. In Section 3, the reinvigorated link between novae and type Ia SNe is discussed. In Sections 4, 5, and 6 the Galactic, extragalactic, and the new population of 'rapid recurrent novae' are presented.

\footnotetext{
${ }^{1} \mathrm{~A}$ recording of the review talk, and the accompanying slides, may be downloaded from http: / / www. $1 \mathrm{jmu}$. ac.uk/ mjd/talks.html
} 
Finally, in Section 7 the most remarkable and extreme nova yet discovered is featured. It is hoped that this article compliments a pair of recent comprehensive reviews of extragalactic novae $[25,26]$.

\section{Recurrent Novae and the MMRD}

Any nova that has been observed in eruption more than once is referred to as recurrent. Observed recurrence periods currently range between $1 \leq P_{\text {rec }} \leq 98$ yrs [27, 28]. Using M 31 and the Milky Way as examples, the RN population only accounts for a few percent of the known nova population [29]. However, there are clear and substantial selection effects at play with regard to both the RN population size and the range of observed periods [26]. For example, the RN population of the extensively observed Large Magellanic Cloud (LMC) makes up a much larger proportion of the host's overall nova population [30].

The short recurrence periods of the RNe are driven by a combination of two fundamental system parameters. (Most) RNe contain high mass WDs and have high mass accretion rates $(\dot{M})$. The increased surface gravity of a high mass WD means less material is required to meet the ignition criteria for H-burning. The higher $\dot{M}$ the more rapidly that critical mass is acquired.

A high mass WD in a nova system can be acquired by two routes: the zero-age WD mass was high, the WD is therefore most likely to be ONe in composition [31]; or the zero-age WD mass was low(er) and the WD mass has increased over the lifetime of the nova system; here the WD is more likely to be CO (see Section 3). The high $\dot{M}$ is achieved via Roche-lobe overflow of a sub-giant or through the wind of a red giant donor (the symbiotic case) [10], with $\dot{M}$ reaching $\sim 10^{-7} \mathrm{M}_{\odot} \mathrm{yr}^{-1}$. Such systems therefore have orbital periods greater than those of typical $\mathrm{CNe}$ ( $P_{\text {orb }} \gtrsim 1 \mathrm{~d}$ versus $P_{\text {orb }} \sim$ a few hours for CNe [10]). Although, in the most extreme case, mass loss may occur through Roche-lobe overflow of a red giant (see Section 7). The lower ignition masses required by RNe leads to substantially less massive, and hence faster evolving, ejecta than are seen from $\mathrm{CNe}$.

The majority of Galactic RNe follow the picture painted above, with 30\% hosting a sub-giant donor and 50\% with a red giant donor [10]. Yet two systems, T Pyxidis and IM Normae - the "recurrent unusual novae" - seemingly with main sequence donors, lower mass WDs, and short orbital periods, may buck this trend (for a non-complete selection of discussion articles about these unusual RNe see [32, 33, 34]). Figure 1 presents a colour-magnitude diagram (based on [10]) that illustrates how near-IR quiescent photometry is used to determine the donor type in a nova system.

The Maximum Magnitude-Rate of Decline (MMRD) relationship for novae has been with us for almost a century $[38,39]$. Simply, the MMRD states that the brighter the nova (at peak) the more rapidly it fades (optically), and typically takes the form of a power-law between maximum luminosity and decay-rate. For a time, the MMRD enabled the popularisation of novae as extragalactic distance indicators - a potential rival to Cepheid variables. But the large MMRD scatter and the high observational overhead hindered its uptake. More recently, the very concept of the MMRD relationship has been questioned. But even the much heralded Gaia (DR2; [40]) has yet been unable to provide compelling evidence either way for Galactic novae (see [41] versus [42]).

Further afield evidence slowly mounts against the MMRD relationship. First in M 31 [43], and then M 87 [44], a new population of 'faint-fast' novae has been proposed - novae that populate a previously devoid area of the MMRD phase space. Well established nova eruption models (e.g., 


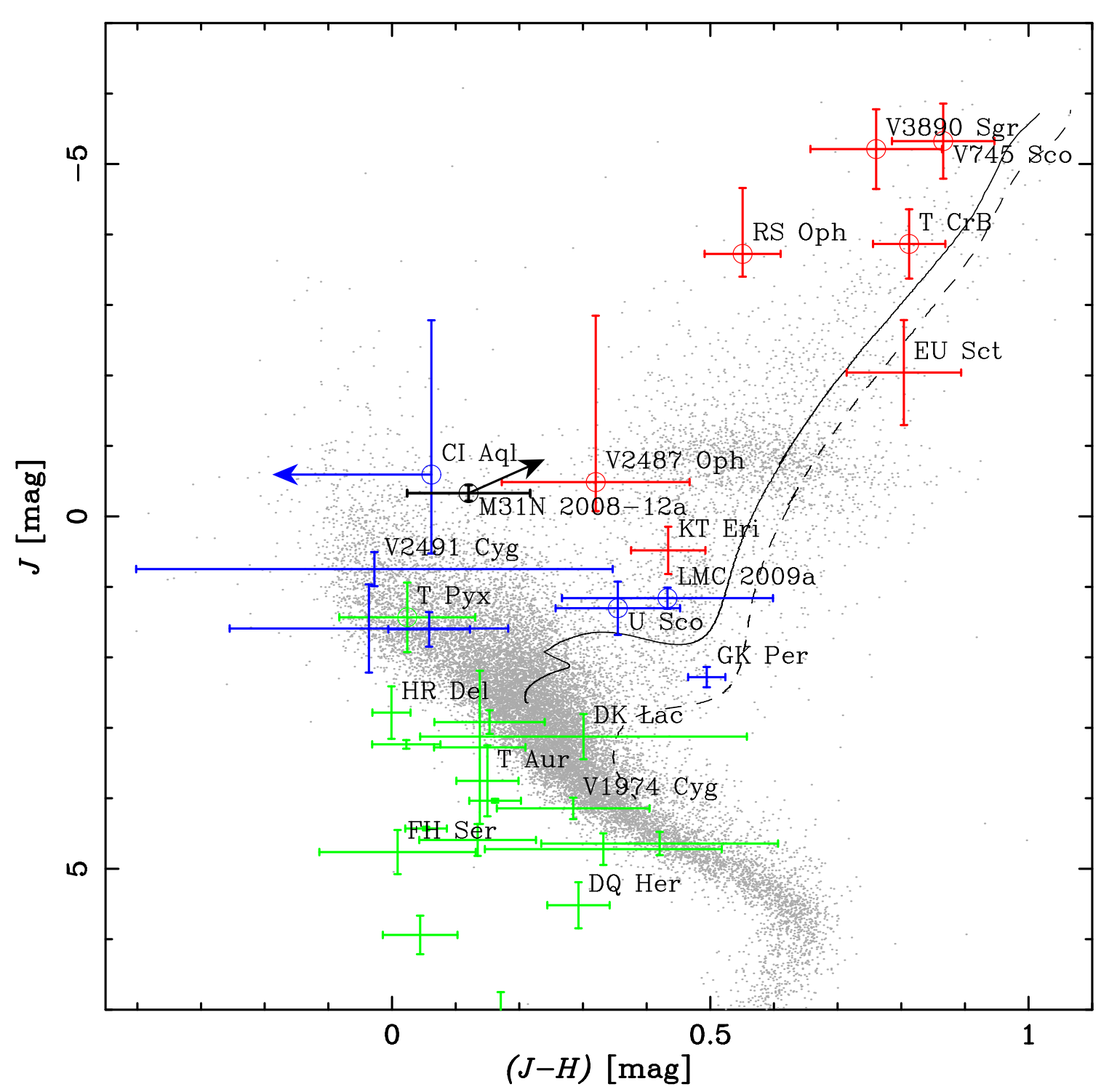

Figure 1: Near-infrared colour-magnitude diagram showing the position of selected (mostly) Galactic novae at quiescence. Red data points indicate those with red giant donors, dark-blue those with sub-giant donors, and green those with main sequence donors. Points additionally circled indicate known recurrent novae. The black data point denotes the position of M31N 2008-12a (see Section 7), with the arrow indicating the evolution during quiescence. The grey data points indicate stars with parallax and photometric errors $<10 \%$ extracted from the Hipparcos catalogue [35] cross-correlated with 2MASS [36], and are included for informative purposes only. The dashed black line and the solid black lines represent evolutionary tracks of 1 and $1.4 \mathrm{M}_{\odot}$ Solar composition stars, respectively [37]. An updated version of Figure 1 from [10]. 
[24]) allow us to interpret the positioning of a nova in the MMRD phase space. The traditional MMRD relationship spans from bright-fast novae that contain high mass WDs accreting at a low rate, to faint-slow novae with low mass WDs and high $\dot{M}[26]^{2}$. The faint-fast novae, therefore, arise from high mass WDs with high $\dot{M}$. Many of the eight long orbital period Galactic RNe (see Section 4) can be classified as faint-fast novae, but to-date, only one (see Section 7) of their faint-fast M 31 and M 87 counterparts has been observed to recur. Recently, [26] proposed that the apparently empty bright-slow MMRD phase space - expected to be occupied by low mass WDs with low $\dot{M}-$ might be occupied by 'SPRITES' (eSPecially Red Intermediate-luminosity Transient Events; [45]), which populate the infrared luminosity space between novae and SNe. This led [26] to propose that exploration of a 'Bolometric MMRD' could have the potential to restore the usefulness of the MMRD.

\section{Helium Flashes and SN Ia Progenitors}

Nova have long been proposed as a potential progenitor pathway along the single-degenerate route toward SN Ia explosions (see, for e.g., [46, 47, 48, 49]). But two barriers have traditionally stood in their way.

The first is simply whether there are enough novae to make a significant enough contribution to the menagerie of potential SN Ia progenitors - and this remains an open question (see, for e.g., $[29,50,51,52])$. Yet novae are probably the most luminous of all pre-SN Ia systems, possibly even during quiescence. Thus nova populations can be studied well beyond the heavily biased constraints of the Milky Way, and the size of their contribution to the SN Ia rate, and any dependance upon host morphology or star formation history, can in principle be determined observationally.

The second barrier relates to the WD mass and how it changes with a nova eruption. For an accreting WD to reach the Chandrasekhar mass it must, of course, increase in mass with time. Each nova eruption can only ever eject a proportion $(<100 \%)$ of the accreted envelope. Some of the accreted hydrogen must remain on the surface to fuel the on-going nuclear burning that is observed as the super-soft X-ray source (SSS). However, if a large quantity of the WD core material mixes with the accreted material (see, for e.g., [53]) the ejecta mass can, in principle, be larger than the critical (ignition) mass - causing the WD mass to decrease. The most recent theoretical studies of nova eruptions - or H-flashes - agree that the accretion efficiency $\eta$ over the course of an eruption cycle is $>0$ (see, for e.g., $[8,23,24,54,55,56,57,58]$ ), i.e. the WD mass increases from one $\mathrm{H}$-flash to the next.

Each H-flash deposits its waste products - in this case $\mathrm{He}$ - onto the WD core (see, for e.g., [59]). As time progresses, this leads to a growing He layer on the WD. Just as for the accreted $\mathrm{H}$ layer, once the He layer attains enough mass, nuclear burning (via the triple- $\alpha$ process) will ensue within the He layer. Initial simulations of such He-flashes indicated that they violently ejected all accreted material from the WD usually along with a substantial proportion of mixed in core material (see, e.g., [60, 61]. Whether H-flashes allowed WD growth or not, it seemed the Heflashes ultimately whittled away the WD.

\footnotetext{
${ }^{2}$ We note that reliable direct measurements or estimates of the WD mass are only available for a small proportion of all novae. In general the WD mass is inferred from observational properties such as the speed class and independently from the X-ray properties.
} 
But, the first study to simulate a long series of He-flashes (each punctuated by $\sim 100 \mathrm{H}$-flashes) made an important discovery [23]. When the WD core (and He layer) temperature was tracked through the evolution of these flashes it was seen to increase [23, 62]. After a number of He-flashes the core temperature had increased sufficiently to, at first, lessen the degeneracy of the He layer, and ultimately remove it. As such, these later He-flashes did not result in TNRs and would not eject material from the system. Instead, the later He-flashes simply convert He to $\mathrm{C} / \mathrm{O}$ and deposit that directly on the WD core $[23,62]$. Ultimately, the WD mass does grow with time - although initially it may decrease until the WD is sufficiently heated. The potential of novae, and particularly the $\mathrm{RNe}$, as SN Ia progenitors was re-ignited.

On this subject, we also refer the reader to the contribution in these proceedings from Sumner Starrfield and collaborators.

\section{Galactic Recurrent Novae}

The ten known Galactic RNe are by far (with the exception of perhaps M31N 2008-12a; see Section 7) the best studied. An extremely comprehensive compilation of their observational properties and history was published in [63]. The Galactic RNe naturally separate into three distinct classes: the symbiotic RNe, the five (four confirmed; V2487 Ophiuchi suspected) with red giant donors, often referred to as the RS Ophiuchi-class; the three with sub-giant donors, or the UScorpiiclass; and the TPyxidis-class, those with short CN-like orbital periods. We summarise some of the properties of these novae in Table 1, which draws from data published in [10, 26, 50, 63, 64].

The population of known Galactic RNe must clearly suffer from (in many cases) severe selection effects and biases. At the time of writing, the shortest inter-eruption period yet seen is the eight years that elapsed between the 1979 and 1987 eruptions of U Sco - whereas numerous examples of much shorter recurrence periods have been seen extragalactically (see Sections 5 and 6). At the other end of the scale, the longest inter-eruption period is the 98 years between the two known eruptions of V2487 Oph [28] - an upper limit that must surely only be related to the availability of historical observations. In a century's time, I firmly predict that the longest known inter-eruption period will be $\sim 200$ yrs.

While there are only ten confirmed Galactic RNe, there are almost as many strong candidates (see [50] for a compilation). In all cases these are $\mathrm{CNe}$ (a single eruption) that share many observational properties with an eruption of a RN. There are very strong contenders, such as KT Eridani [65, 66, 67, 68]; and more 'conflicted' cases, such as V2491 Cygni [50, 69, 70, 71, 72]. All of these systems deserve continued study and warrant long-term monitoring to catch the expected, or proposed, second eruptions.

In an attempt to quantify the number of RNe that masquerade as $\mathrm{CNe}$, [50] compiled a set of observational properties of RNe. These include: low amplitude eruptions (due to the high quiescent luminosity of an evolved donor and a high $\dot{M}$ — therefore bright — accretion disk); long orbital periods (required to physically accommodate the enlarged radius of an evolved donor); NIR bright at quiescent (again a property of the donor); high velocity ejecta (due to the high surface gravity, and hence high escape velocity, of the WD); high excitation lines (visible due to the energetics of the eruption and the low optical depth of the low mass ejecta); and a light curve plateau (proposed by many, including $[19,73]$, to be due to unveiling of the surviving high $\dot{M}$ accretion disk as the 
Table 1: Selected properties of the ten known Galactic recurrent novae.

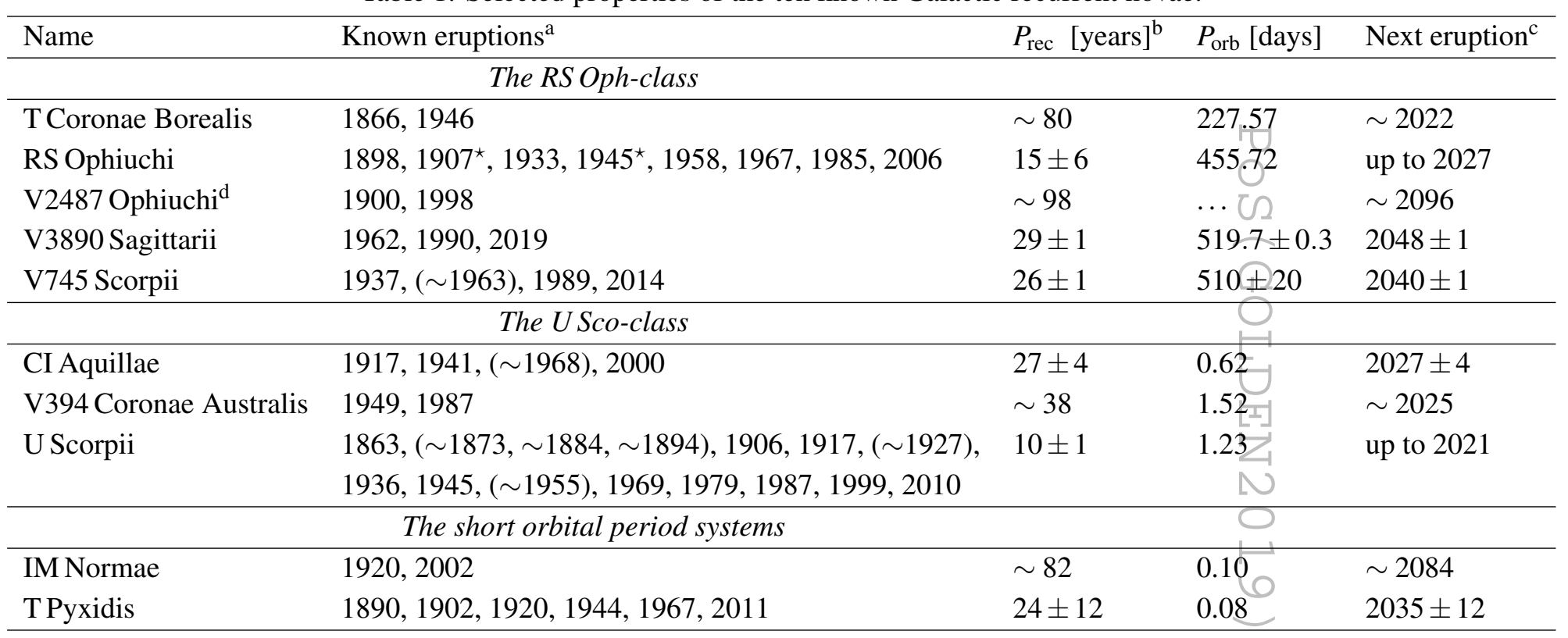

This table is based upon data compiled within $[10,26,50,63,64]$ and references therein.

a Suspected eruptions indicated by $\star$; assumed eruptions (by this author) indicated by parentheses. A 1945 eruption of RS Oph was long suspected, [74] presented evidence in support that eruption occurring during a seasonal gap.

${ }^{\mathrm{b}}$ With the exception of T Pyx, the average inter-eruption times are presented based on the assumption that eruptions (i.e. $\dot{M}$ ) are relatively uniformly spaced (see Section 7). For T Pyx we simply report the mean observed $P_{\text {rec }}$.

${ }^{\mathrm{c}}$ Predicted dates of next eruptions are based on the assumed $P_{\mathrm{rec}}$ for each system. At the time of writing, RS Oph and U Sco are already within their predicted windows.

d There are no orbital period data for V2487 Oph available. Based on quiescent NIR photometry, [10] identified the donor as a low-luminosity red giant (possibly red-clump) star and hence placed V2487 Oph in the RS Oph class. 
photosphere recedes back toward the WD). A key prediction from [50] is that around a third of all Galactic novae may be recurrent (formally $10 \leq P_{\text {rec }} \leq 100$ yrs; A. Pagnotta, priv. comm.).

\section{Extragalactic Recurrent Novae}

There are currently over 1100 nova candidates within $\mathrm{M} 31^{3}$ [75]. Indeed there are more nova candidates in M31 than known novae in all other galaxies (the Milky Way included) combined. By virtue of its high nova eruption rate [3] combined with its closeness, M31 has become the preferred laboratory for the study of nova populations [26]. But M 31 is far from ideal, its large angular size has led to the majority of surveys (particularly historic campaigns) focussing only on the bulge or the brighter inner regions [26]. This is far from surprising, with the nova population of a galaxy broadly following the light, additional fields away from the centre only provide diminishing returns. Of course this places substantial spatial (and temporal) biases on any population studies using those novae. Viewed from the Milky Way, M31 is relative close to being edge-on [76], which results in large internal extinction uncertainties $[3,77]$ and severely challenges efforts to disentangle the bulge and disk populations within that host [3]. However, [3] found significant evidence for separate bulge and disk nova populations within M31. More recently [78], utilising wide-field high-cadence surveys of M 31, reported the discovery of two novae associated with the Giant Stellar Stream to the south of M31 (see [79]).

Of those 1100 M 31 nova candidates, in excess of 200 have now been spectroscopically confirmed [80, 81]. Within that subset lie eighteen known RNe. While a small number of the M 31 RNe have been known or suspected for some time, the first comprehensive catalogue of these systems was published by [29], who found 16. An additional three M31 RNe have been added [82, 83, 84, 85], and one recanted [86]. A completeness analysis (of the 16) [29] indicated that the detection efficiency of M31 RNe - i.e. the probability of detecting at least a second eruption may be as low as $10 \%$. Subsequently, as many as 1 in 3 M 31 nova eruptions may be from a RN (here, formally, $1 \leq P_{\text {rec }} \leq 100$ yrs).

Add in to the mix the four known RNe in the $\operatorname{LMC}[26,30]$ and that's it - 32 known $\mathrm{RNe}$ spanning just three galaxies. In most other Local Group galaxies the observations are just too sparse (e.g., M33; [87]) or the nova rate too low (e.g., SMC; [30, 88]) to have yet reached a good probability of detecting a RN. Beyond the Local Group, M 81 [89] and M 87 [4] are the next best sampled hosts, but they too are yet to produce a published RN.

The first extragalactic nova progenitor, or quiescent system, that of M31N 2007-12b, was recovered in M 31 using archival Hubble Space Telescope (HST) ACS/WFC data [90]. That candidate (still unconfirmed) RN was shown to harbour a red giant donor. Subsequently, [91] demonstrated that all novae with red giant donors (which Galactically are dominated by known RNe; [10]; see Figure 1) are accessible to HST within the Local Group. The first extragalactic survey for quiescent novae was conducted within M 31 by [51] who produced a catalogue of eleven progenitor systems, each hosting a red giant donor. The subsequent statistical analysis [52] reported that 30\% of M 31 novae may contain a red giant donor. Moreover, that population of potential RNe was strongly associated with the (young) disk population of M31. The [52] result was indeed statistically consistent

\footnotetext{
${ }^{3}$ For the most complete, and up to date, catalogue, see: http://www.mpe.mpg.de/ m31novae/index. php
} 
with all the red giant novae being disk novae. While far from proven, a potential consequence might be that novae (with red giant donors) only contribute to the SN Ia rate in late-type galaxies.

\section{Rapid Recurrent Novae}

As a WD in a nova system approaches the Chandrasekhar mass, $P_{\text {rec }}$ should decrease (see, for e.g., [24]). Theory shows us that on the cusp of breaching that limit $P_{\text {rec }}$ may be as low as two months $[22,23]$. But when we look at the Galactic $\mathrm{RN}$ population the most rapidly recurring system is USco with a mean inter-eruption time scale of only $10 \mathrm{yrs}$ - close to two orders of magnitude slower than the predicted extreme. So if a nova WD really can be grown toward the Chandrasekhar mass, where are the more rapidly recurring systems?

If one examines the RN populations of the LMC and M 31 there is a striking difference between the RNe from those hosts and those from the Milky Way. Of the four known LMC RNe, one (LMCN 1968-12a) has $P_{\text {rec }}=6.2 \pm 1.2$ yrs [92] - almost twice as fast as U Sco. But of the eighteen M31 RNe (see Section 5) half have recurrence periods similar to or shorter than U Sco! The existence of this quick-fire population of RNe led [26] to dub all systems with $P_{\text {rec }} \leq 10 \mathrm{yrs}$ 'rapid recurrent novae' (RRNe). In fact all new M 31 RNe discovered since 1984 - of which there are seven - are RRNe. Figure 2 illustrates the distribution of RN recurrence periods within the Milky Way, M31, and the LMC. Is this difference due to variation between the stellar (and therefore nova) populations of these galaxies, related possibly to differing star formation histories and metallicities, or are observational select effects at play?

So where are the Galactic RRNe? If they exist, they are most likely hiding in plain sight. RRNe must contain high mass WDs accreting at an elevated rate [24]. Thus RRNe should be faint-fast novae (see Section 2). RRNe are therefore particularly challenging to detect, let alone classify. Galactically, perhaps somewhat ironically, the largest background signal to RRNe may actually be the dwarf novae (DNe; see [93] for an authoritative overview). Unfortunately, most DNe are not followed-up sufficiently (i.e. optical spectroscopy and X-ray observations, and in many cases not even photometrically) to be able to distinguish them from a low amplitude, quasi-short-periodic, nova eruption - a RRN eruption.

But we have been able to successfully detect and follow-up RRNe beyond the Milky Way, particularly in M31 (e.g., [27]), because M31 (and the LMC) cover relatively small regions of the sky. The advent of large area detectors coupled with high cadence observations, and the invaluable input from the amateur astronomy community, have enabled M 31 to be covered in both unprecedented depth and cadence over the last decade, or so. This change in observing strategy and capability led directly to the discovery of RRNe. Rapid response follow-up facilities, such as the Liverpool Telescope [94] and the Neil Gehrels Swift Observatory [95, 96], have permitted the study and confirmation of those RRN candidates ${ }^{4}$.

We stand on the precipice of a new era of all-sky high cadence surveys. The SSS-phase of $\mathrm{RRNe}$ (and novae in general) is perhaps their most unique 'fingerprint'. The recently launched eROSITA (see, for e.g., [97]) could be vital for detecting new RRNe in the Milky Way. Optical

\footnotetext{
${ }^{4}$ It may also be worthwhile noting that (M31) DNe are not visible above from unresolved surface brightness of M 31 with the typical seeing experienced by ground-based observations.
} 


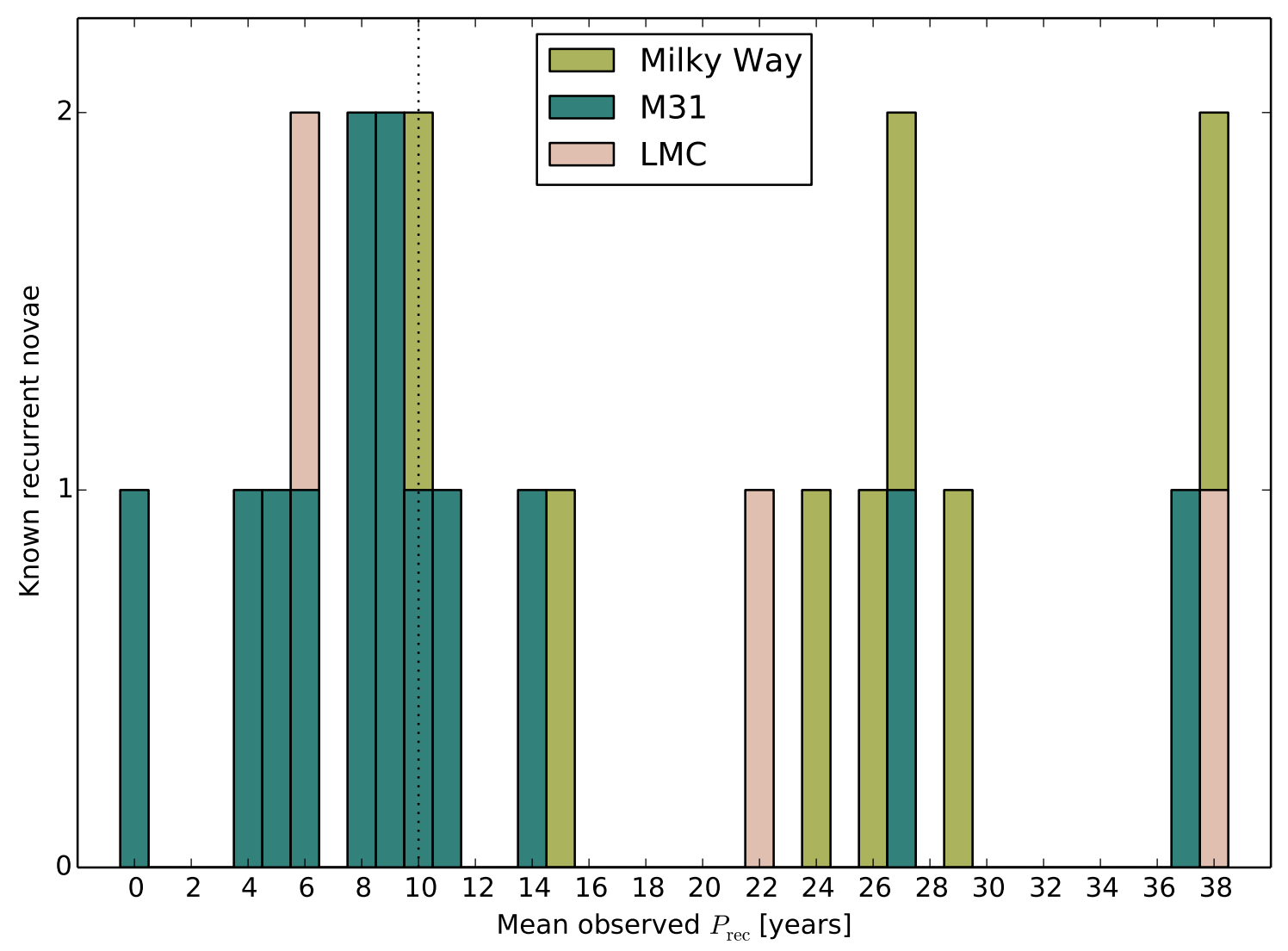

Figure 2: The distribution of the mean inter-eruption periods of the known recurrent novae (the plot only shows systems with $P_{\text {rec }} \leq 40 \mathrm{yrs} ; 9 \mathrm{RNe}$ with longer periods are not shown). The dotted vertical line demarks the realm of the rapid recurrent novae $\left(P_{\mathrm{rec}} \leq 10 \mathrm{yrs}\right)$. Adapted and updated from Figure 3 of [26].

facilities, such as the Large Synoptic Survey Telescope (LSST; [98]) should dramatically alter the landscape for transient object astronomy. LSST, for example, will be sensitive to RRN eruptions in the Milky Way and Magellanic Clouds. But for optical discoveries of RRNe, the problem will ultimately lie with the availability of fast enough spectroscopic (or soft X-ray) follow-up. While many follow-up facilities complementing LSST and its contemporaries have been or are being planned and built (e.g., [99]) there are unlikely to be anywhere near enough to classify all new transient detections. So for any novae, not specifically RRNe, to reap any benefit from LSST et al., the value of nova science to all of astronomy requires substantial promotion. Right now, one RRN system in particular may be key.

\section{M31N 2008-12a}

"In all your travels, have you ever seen a star go supernova?" [100]. Any denizen of the Andromeda Galaxy, particularly the region surrounding the unassumingly named binary system RX J0045.4+4154, may have borne witness to a nearby star undergoing a fantastic increase in 
luminosity around once a (Terran) year. Perhaps this system, may even be central to the culture of those nearby 'Andromedans'. Little might they know that, given the right conditions, and the right composition, RX J0045.4+4154 may one day explode as a SN Ia, and potentially — unfortunately — wipe out all in the immediate vicinity.

RX J0045.4+4154 itself usually goes by its more familiar (yet equally cumbersome) name M31N 2008-12a - it is the prototype RRN, and the most extreme example of a nova yet discovered. Of all known novae, probably of all known stellar systems, is the most likely to explode first as a SN Ia. And, given the distance to M 31, it probably already has.

The first observed eruption of '12a' (by humans) took place on Christmas Day 2008 [101]. Since then, it has been observed in eruption every single year, most recently in November 2019 [102]. Although suspected beforehand, the true nature of 12a was not confirmed until the 2013 eruption $[27,103,104]$. At the time, annual nova eruptions were completely unprecedented. Although a small number of RRNe had already been found in M31, none had been studied in any detail. Until then the most rapidly recurring (and therefore extreme) $\mathrm{RN}$ was generally accepted to be U Sco.

$12 \mathrm{a}$ is powered by the combination of the most massive WD known (predicted via some models to be $\left.\simeq 1.38 \mathrm{M}_{\odot} ;[58]\right)$ accreting, stably, at an exceptionally high rate in the region of $(0.6 \lesssim \dot{M} \lesssim 1.4) \times 10^{-6} \mathrm{M}_{\odot} \mathrm{yr}^{-1}$ [105]. Together, this drives eruptions with a mean $P_{\text {rec }}=0.99 \pm$ $0.02 \mathrm{yrs}$ [26]. The high accretion rate has been proposed to be due to a Roche-lobe overflowing red giant (or red clump) donor [105]. At present, the composition of the 12a WD has not been confirmed. But, HST STIS FUV spectra taken just $3 \mathrm{~d}$ after the 2015 eruption showed no indication of $\mathrm{Ne}$ in the ejecta ${ }^{5}$ [108]. This implies - but does not confirm — that the 12a WD is CO and that the system is building toward a SN Ia explosion. A very conservative prediction suggested such an event will occur in $<20$ kyr [105].

Comprehensive overviews and summaries of $12 \mathrm{a}$ and its eruptions are presented in [26, 109, 110]. Selected highlights, that illustrate the extreme nature of the system include: low mass ejecta with low optical luminosity, consistent with high $\dot{M}$, high mass WD, and ultra-short $P_{\text {rec }}$ models of TNRs [24, 58]; ejecta deceleration as they shock the pre-existing disk/donor wind [105, 109]; the highest SSS effective temperature seen in a nova ( $T_{\text {eff }} \sim 120 \mathrm{eV}$; [109]); detection of significant high-amplitude SSS variability [19]; remarkable similarity between the 2013-2015 and 2017-2019 eruptions [109, 111]; detection of short-lived high velocity, collimated (jet-like), outflows [108, 109]; and recovery of the progenitor from archival HST data [27, 105].

But the most remarkable aspect of 12a is the effect it has had upon the local environment. The system is surrounded by a vast shell-like nebula that is the remnant of all the eruptions that have enabled the WD to grow toward the Chandrasekhar mass $[112,113]$. This 'nova super-remnant' (NSR) has a major axis of $134 \mathrm{pc}$ and consists of $\sim 10^{5-6} \mathrm{M}_{\odot}$ of swept up ISM [113]. Hydrodynamical simulations of 100,000 periodic (annual) 12a-like eruptions clearly demonstrated that repeated RN eruptions are capable of producing such a phenomenon [113].

Any nova system, as it grows toward the Chandrasekhar mass, should therefore be surrounded by a similar NSR. It is clear that the size of these objects will depend upon parameters such as the zero-age and current WD mass, the accretion history, and surrounding ISM density. But fac-

\footnotetext{
${ }^{5} \mathrm{~A}$ Ne over-abundance is a signature of an $\mathrm{ONe}$ WD [106, 107].
} 
tors such as metalicity, donor type, and even line-of-sight inclination may affect the observational properties. NSRs clearly deserve substantial further theoretical and observational study. The hunt is already on to find further examples.

\section{Summary}

As a sub-set of all novae, $\mathrm{RNe}$ present generally the more extreme system and eruption parameters. Their short inter-eruption periods permit detailed study of repeated eruptions from the same system. With growing WDs, RNe are strong candidates for a single-degenerate SN Ia pathway. In general, the shorter the recurrence period the closer a given system is to reaching the Chandrasekhar mass. Currently, the known RN population is beset by substantial selection effects, particularly Galactically. But those challenges can be, and are being, overcome. By doing so the contribution of novae to the SN Ia rate can be quantified.

Recurrent nova super-remnants present a new opportunity to not only identify previously unknown RNe and RRNe, but potentially to locate any 'extinct' systems — former RNe, where the donor mass reservoir has been depleted. But perhaps more intriguingly, should a RN explode as a SN Ia - due to its vast scale - the NSR will provide a clear and persistent signpost to the progenitor-type of that SN Ia. By its very nature, NSR formation will have also pre-prepared the local environment in which the SN Ia will explode. Importantly, if the 12a NSR is common, such $\mathrm{RN}$ eruptions will have moved around $10^{6} \mathrm{M}_{\odot}$ of (predominantly) hydrogen away from the site of the imminent SN Ia, and it will take centuries before an expanding SN Ia shock interacts with that material. RNe and NSRs may therefore provide a solution to the single-degenerate SN Ia 'paradox'; where is the hydrogen?

Of course, the big unanswered question is whether M31N 2008-12a and its super-remnant is a rare, or even unique, phenomenon; or is it just the tip of the iceberg?

\section{Acknowledgments}

The study of novae, Galactically and extragalactically, is heavily indebted to the continuing support provided by the amateur astronomy community. I would therefore like to take this opportunity to pass on my thanks to all those who contribute to the work of the nova community, and astronomy in general. That includes those affiliated with the AAVSO, the BAA, and the VSOLJ, from which the 12a work in particular draws heavily. I would like to thank the Scientific and Local Organising Committees of The Golden Age of Cataclysmic Variables and Related Objects V, particularly Franco Giovannelli and Francesco Reale, for their kind invitation to present a review talk on Recurrent Novae. I would also like to extend my thanks to the organisers for still being able to accommodate me (remotely) once it became clear that I was unable to travel to the workshop. I would also like to thank my friend and collaborator Martin Henze for useful comments on this manuscript. I must acknowledge partial funding from the UK Science and Technology Facilities Council (STFC), who supported much of my own work over the past six years. Finally, I would like to pass my thanks to the referee of this review, Sumner Starrfield, whose comments help improved this work. 


\section{References}

[1] M. M. Kasliwal, Bridging the gap : elusive explosions in the local universe, Ph.D. thesis, California Institute of Technology, Jan, 2011.

[2] A. W. Shafter, The Galactic Nova Rate Revisited, ApJ 834 (2017) 196 [1606.02358].

[3] M. J. Darnley, M. F. Bode, E. Kerins, A. M. Newsam, J. An, P. Baillon et al., Classical novae from the POINT-AGAPE microlensing survey of M31 - II. Rate and statistical characteristics of the nova population, MNRAS 369 (2006) 257 [astro-ph/ 0509493 ].

[4] M. M. Shara, T. F. Doyle, T. R. Lauer, D. Zurek, J. D. Neill, J. P. Madrid et al., A Hubble Space Telescope Survey for Novae in M87. I. Light and Color Curves, Spatial Distributions, and the Nova Rate, ApJS 227 (2016) 1 [1602.00758].

[5] A. W. Shafter, A. Kundu and M. Henze, On the Nova Rate in M87, Research Notes of the American Astronomical Society 1 (2017) 11 [1712.05818].

[6] A. Tajitsu, K. Sadakane, H. Naito, A. Arai and W. Aoki, Explosive lithium production in the classical nova V339 Del (Nova Delphini 2013), Nature 518 (2015) 381 [1502 . 055 98].

[7] L. Izzo, P. Molaro, P. Bonifacio, M. Della Valle, Z. Cano, A. de Ugarte Postigo et al., Beryllium detection in the very fast nova ASASSN-16kt (V407 Lupi), MNRAS 478 (2018) 1601 [1802.05896].

[8] S. Starrfield, M. Bose, C. Iliadis, W. R. Hix, C. E. Woodward and R. M. Wagner, Carbon-Oxygen Classical Novae are Galactic ${ }^{7}$ Li Producers as well as Potential Supernova Ia Progenitors, arXiv e-prints (2019) arXiv:1910.00575 [1910.00575].

[9] J. José, Stellar Explosions: Hydrodynamics and Nucleosynthesis. CRC/Taylor and Francis, Boca Raton, FL, USA, 2016, 10.1201/b19165.

[10] M. J. Darnley, V. A. R. M. Ribeiro, M. F. Bode, R. A. Hounsell and R. P. Williams, On the Progenitors of Galactic Novae, ApJ 746 (2012) 61 [1112 . 2589].

[11] M. F. Bode and A. Evans, eds., Classical Novae, 2nd Edition, vol. 43 of Cambridge Astrophysics Series. Cambridge University Press, Cambridge, Apr., 2008.

[12] P. A. Woudt and V. A. R. M. Ribeiro, eds., Stella Novae: Past and Future Decades, vol. 490 of Astronomical Society of the Pacific Conference Series, (San Francisco), Astronomical Society of the Pacific, Dec., 2014.

[13] S. Starrfield, J. W. Truran, W. M. Sparks and G. S. Kutter, CNO Abundances and Hydrodynamic Models of the Nova Outburst, ApJ 176 (1972) 169.

[14] S. Starrfield, C. Iliadis and W. R. Hix, The Thermonuclear Runaway and the Classical Nova Outburst, PASP 128 (2016) 051001.

[15] D. Prialnik, M. M. Shara and G. Shaviv, The evolution of a slow nova model with a $Z=0.03$ envelope from pre-explosion to extinction, A\&A 62 (1978) 339.

[16] K.-L. Li, B. D. Metzger, L. Chomiuk, I. Vurm, J. Strader, T. Finzell et al., A nova outburst powered by shocks, Nature Astronomy 1 (2017) 697 [1709.00763].

[17] S. N. Shore, Spectroscopy of novae - a user's manual, Bulletin of the Astronomical Society of India 40 (2012) 185 [1211. 3176$].$ 
[18] J. J. Drake and S. Orlando, The Early Blast Wave of the 2010 Explosion of U Scorpii, ApJ 720 (2010) L195 [1007 . 2810].

[19] M. Henze, M. J. Darnley, S. C. Williams, M. Kato, I. Hachisu, G. C. Anupama et al., Breaking the Habit: The Peculiar 2016 Eruption of the Unique Recurrent Nova M31N 2008-12a, ApJ 857 (2018) 68 [1803.00181].

[20] B. E. Schaefer, A. Pagnotta, A. P. LaCluyze, D. E. Reichart, K. M. Ivarsen, J. B. Haislip et al., Eclipses during the 2010 Eruption of the Recurrent Nova U Scorpii, ApJ 742 (2011) 113 [1108.1214].

[21] H. L. Worters, S. P. S. Eyres, G. E. Bromage and J. P. Osborne, Resumption of mass accretion in RS Oph, MNRAS 379 (2007) 1557 [0706.1213].

[22] M. Kato, H. Saio, I. Hachisu and K. Nomoto, Shortest Recurrence Periods of Novae, ApJ 793 (2014) 136 [1404.0582].

[23] Y. Hillman, D. Prialnik, A. Kovetz and M. M. Shara, Growing White Dwarfs to the Chandrasekhar Limit: The Parameter Space of the Single Degenerate SNIa Channel, ApJ 819 (2016) 168 [1508.03141].

[24] O. Yaron, D. Prialnik, M. M. Shara and A. Kovetz, An Extended Grid of Nova Models. II. The Parameter Space of Nova Outbursts, ApJ 623 (2005) 398 [astro-ph/ 0503143 ].

[25] A. W. Shafter, Extragalactic Novae, 2514-3433. IOP Publishing, 2019, 10.1088/2514-3433/ab2c63.

[26] M. J. Darnley and M. Henze, On a century of extragalactic novae and the rise of the rapid recurrent novae, Advances in Space Research (2019) .

[27] M. J. Darnley, S. C. Williams, M. F. Bode, M. Henze, J.-U. Ness, A. W. Shafter et al., A remarkable recurrent nova in $M$ 31: The optical observations, A\&A 563 (2014) L9 [1401.2905].

[28] A. Pagnotta, B. E. Schaefer, L. Xiao, A. C. Collazzi and P. Kroll, Discovery of a Second Nova Eruption of V2487 Ophiuchi, AJ 138 (2009) 1230 [0908 .2143].

[29] A. W. Shafter, M. Henze, T. A. Rector, F. Schweizer, K. Hornoch, M. Orio et al., Recurrent Novae in M31, ApJS 216 (2015) 34 [1412.8510].

[30] A. W. Shafter, Photometric and Spectroscopic Properties of Novae in the Large Magellanic Cloud, AJ 145 (2013) 117 [1302.6285].

[31] J. Iben, I. and A. V. Tutukov, On the evolution of close binaries with components of initial mass between 3 M and 12 M., ApJS 58 (1985) 661.

[32] B. E. Schaefer, A. Pagnotta and M. M. Shara, The Nova Shell and Evolution of the Recurrent Nova T Pyxidis, ApJ 708 (2010) 381 [0906.0933].

[33] J. Patterson, A. Oksanen, J. Kemp, B. Monard, R. Rea, F.-J. Hambsch et al., T Pyxidis: death by a thousand novae, MNRAS 466 (2017) 581 [1603.00291].

[34] P. Godon, E. M. Sion, R. E. Williams and S. Starrfield, The Long-term Secular Mass Accretion Rate of the Recurrent Nova T Pyxidis, ApJ 862 (2018) 89 [1806.06059].

[35] M. A. C. Perryman, L. Lindegren, J. Kovalevsky, E. Hoeg, U. Bastian, P. L. Bernacca et al., The HIPPARCOS Catalogue, A\&A 323 (1997) L49.

[36] M. F. Skrutskie, R. M. Cutri, R. Stiening, M. D. Weinberg, S. Schneider, J. M. Carpenter et al., The Two Micron All Sky Survey (2MASS), AJ 131 (2006) 1163. 
[37] A. Pietrinferni, S. Cassisi, M. Salaris and F. Castelli, A Large Stellar Evolution Database for Population Synthesis Studies. I. Scaled Solar Models and Isochrones, ApJ 612 (2004) 168 [astro-ph/0405193].

[38] E. P. Hubble, A spiral nebula as a stellar system, Messier 31., ApJ 69 (1929) 103.

[39] D. B. Mclaughlin, The Relation between Light-Curves and Luminosities of Novae, PASP 57 (1945) 69.

[40] Gaia Collaboration, A. G. A. Brown, A. Vallenari, T. Prusti, J. H. J. de Bruijne, C. Babusiaux et al., Gaia Data Release 2. Summary of the contents and survey properties, A\&A 616 (2018) A1 [1804.09365].

[41] B. E. Schaefer, The distances to Novae as seen by Gaia, MNRAS 481 (2018) 3033 [1809.00180].

[42] P. Selvelli and R. Gilmozzi, A UV and optical study of 18 old novae with Gaia DR2 distances: mass accretion rates, physical parameters, and MMRD, A\&A 622 (2019) A186 [1903. 05868 ].

[43] M. M. Kasliwal, S. B. Cenko, S. R. Kulkarni, E. O. Ofek, R. Quimby and A. Rau, Discovery of a New Photometric Sub-class of Faint and Fast Classical Novae, ApJ 735 (2011) 94 [1003.1720].

[44] M. M. Shara, T. Doyle, T. R. Lauer, D. Zurek, E. A. Baltz, A. Kovetz et al., A Hubble Space Telescope Survey for Novae in M87. II. Snuffing out the Maximum Magnitude-Rate of Decline Relation for Novae as a Non-standard Candle, and a Prediction of the Existence of Ultrafast Novae, ApJ 839 (2017) 109 [1702.05788].

[45] M. M. Kasliwal, J. Bally, F. Masci, A. M. Cody, H. E. Bond, J. E. Jencson et al., SPIRITS: Uncovering Unusual Infrared Transients with Spitzer, ApJ 839 (2017) 88 [1701.01151].

[46] J. Whelan and I. Iben, Jr., Binaries and Supernovae of Type I, ApJ 186 (1973) 1007.

[47] I. Hachisu, M. Kato, K. Nomoto and H. Umeda, A New Evolutionary Path to Type IA Supernovae: A Helium-rich Supersoft X-Ray Source Channel, ApJ 519 (1999) 314 [astro-ph/9902303].

[48] I. Hachisu, M. Kato and K. Nomoto, A Wide Symbiotic Channel to Type IA Supernovae, ApJ 522 (1999) 487 [astro-ph/9902304].

[49] W. Hillebrandt and J. C. Niemeyer, Type IA Supernova Explosion Models, Annual Review of Astronomy and Astrophysics 38 (2000) 191 [astro-ph/0006305].

[50] A. Pagnotta and B. E. Schaefer, Identifying and Quantifying Recurrent Novae Masquerading as Classical Novae, ApJ 788 (2014) 164 [1405.0246].

[51] S. C. Williams, M. J. Darnley, M. F. Bode, A. Keen and A. W. Shafter, On the Progenitors of Local Group Novae. I. The M31 Catalog, ApJS 213 (2014) 10 [1405.4874].

[52] S. C. Williams, M. J. Darnley, M. F. Bode and A. W. Shafter, On the Progenitors of Local Group Novae. II. The Red Giant Nova Rate of M31, ApJ 817 (2016) 143 [1512. 0408 8].

[53] J. Casanova, J. José and S. N. Shore, Two-dimensional simulations of mixing in classical novae: The effect of white dwarf composition and mass, A\&A 619 (2018) A121 [1807.10646].

[54] A. Kovetz and D. Prialnik, Accretion onto a 1.4 solar mass white dwarf: Classical nova, recurrent nova, or supernova?, ApJ 424 (1994) 319.

[55] D. Prialnik and A. Kovetz, An extended grid of multicycle nova evolution models, ApJ 445 (1995) 789. 
[56] S. Starrfield, C. Iliadis, F. X. Timmes, W. R. Hix, W. D. Arnett, C. Meakin et al., Theoretical studies of accretion of matter onto white dwarfs and the single degenerate scenario for supernovae of Type Ia, Bulletin of the Astronomical Society of India 40 (2012) 419 [1210 . 6086 ].

[57] Y. Hillman, D. Prialnik, A. Kovetz and M. M. Shara, Observational signatures of SNIa progenitors, as predicted by models, MNRAS 446 (2015) 1924 [1411.0382].

[58] M. Kato, H. Saio and I. Hachisu, Multi-wavelength Light Curve Model of the One-year Recurrence Period Nova M31N 2008-12A, ApJ 808 (2015) 52 [1506. 05364 ].

[59] M. Kato, H. Saio and I. Hachisu, A Millennium-long Evolution of the 1 yr Recurrence Period Nova-Search for Any Indication of the Forthcoming He Flash, ApJ 844 (2017) 143 [1706.08654].

[60] I. Idan, N. J. Shaviv and G. Shaviv, The fate of a WD accreting H-rich material at high accretion rates, MNRAS 433 (2013) 2884.

[61] G. Newsham, S. Starrfield and F. X. Timmes, Evolution of Accreting White Dwarfs: Some of Them Continue to Grow, in Stellar Novae: Past and Future Decades, P. A. Woudt and V. A. R. M. Ribeiro, eds., vol. 490 of Astronomical Society of the Pacific Conference Series, p. 287, Dec., 2014, 1303.3642 .

[62] Y. Hillman, M. Shara, D. Prialnik and A. Kovetz, Multi-outburst nova modeling \& where models meet observations, Advances in Space Research (2019).

[63] B. E. Schaefer, Comprehensive Photometric Histories of All Known Galactic Recurrent Novae, ApJS 187 (2010) 275 [0912.4426].

[64] G. C. Anupama, The Recurrent Nova Class of Objects, in RS Ophiuchi (2006) and the Recurrent Nova Phenomenon, A. Evans, M. F. Bode, T. J. O’Brien and M. J. Darnley, eds., vol. 401 of Astronomical Society of the Pacific Conference Series, pp. 31-41, 2008.

[65] R. Hounsell, M. F. Bode, P. P. Hick, A. Buffington, B. V. Jackson, J. M. Clover et al., Exquisite Nova Light Curves from the Solar Mass Ejection Imager (SMEI), ApJ 724 (2010) 480 [1 009.1737 ].

[66] R. Jurdana-Šepić, V. A. R. M. Ribeiro, M. J. Darnley, U. Munari and M. F. Bode, Historical light curve and search for previous outbursts of Nova KT Eridani (2009), A\&A 537 (2012) A34 [1110.4637].

[67] V. A. R. M. Ribeiro, M. F. Bode, M. J. Darnley, R. M. Barnsley, U. Munari and D. J. Harman, Morpho-kinematical modelling of Nova Eridani 2009 (KT Eri), MNRAS 433 (2013) 1991 [1305.3834].

[68] U. Munari, E. Mason and P. Valisa, The narrow and moving HeII lines in nova KT Eridani, A\&A 564 (2014) A76 [1403.3284].

[69] K. L. Page, J. P. Osborne, P. A. Evans, G. A. Wynn, A. P. Beardmore, R. L. C. Starling et al., Swift observations of the X-ray and UV evolution of V2491 Cyg (Nova Cyg 2008 No. 2), MNRAS 401 (2010) 121 [0909.1501].

[70] V. A. R. M. Ribeiro, M. J. Darnley, M. F. Bode, U. Munari, D. J. Harman, I. A. Steele et al., The morphology of the expanding ejecta of V2491 Cygni (2008 N.2), MNRAS 412 (2011) 1701 [1011.2045].

[71] U. Munari, A. Siviero, S. Dallaporta, G. Cherini, P. Valisa and L. Tomasella, An extensive optical study of V2491 Cyg (Nova Cyg 2008 N.2), from maximum brightness to return to quiescence, New Astronomy 16 (2011) 209 [1009.0822]. 
[72] M. J. Darnley, V. A. R. M. Ribeiro, M. F. Bode and U. Munari, On the progenitor system of Nova V2491 Cygni, A\&A 530 (2011) A70 [1104.3482].

[73] I. Hachisu, M. Kato, S. Kiyota, K. Kubotera, H. Maehara, K. Nakajima et al., Optical Light Curves of RS Oph (2006) and Hydrogen Burning Turnoff, in RS Ophiuchi (2006) and the Recurrent Nova Phenomenon, A. Evans, M. F. Bode, T. J. O’Brien and M. J. Darnley, eds., vol. 401 of Astronomical Society of the Pacific Conference Series, (San Francisco), pp. 206-209, Dec., 2008, 0807.1240.

[74] S. Adamakis, S. P. S. Eyres, A. Sarkar and R. W. Walsh, A pre-outburst signal in the long-term optical light curve of the recurrent nova RS Ophiuchi, MNRAS 414 (2011) 2195 [1102.3779].

[75] W. Pietsch, X-ray emission from optical novae in M 31, Astronomische Nachrichten 331 (2010) 187 [0910.3865].

[76] G. de Vaucouleurs and H. G. Corwin, Jr., S Andromedae 1885 - A centennial review, ApJ 295 (1985) 287.

[77] J. J. Dalcanton, M. Fouesneau, D. W. Hogg, D. Lang, A. K. Leroy, K. D. Gordon et al., The Panchromatic Hubble Andromeda Treasury. VIII. A Wide-area, High-resolution Map of Dust Extinction in M31, ApJ 814 (2015) 3 [1509.06988].

[78] M. J. Darnley, A. M. Newsam, K. Chinetti, I. D. W. Hawkins, A. L. Jannetta, M. M. Kasliwal et al., AT 2016dah and AT 2017fyp: the first classical novae discovered within in tidal stream, Submitted, for publication in MNRAS (2020) .

[79] R. Ibata, M. Irwin, G. Lewis, A. M. N. Ferguson and N. Tanvir, A giant stream of metal-rich stars in the halo of the galaxy M31, Nature 412 (2001) 49 [a st ro-ph / 0107090 ].

[80] A. W. Shafter, M. J. Darnley, K. Hornoch, A. V. Filippenko, M. F. Bode, R. Ciardullo et al., $A$ Spectroscopic and Photometric Survey of Novae in M31, ApJ 734 (2011) 12 [1104.0222].

[81] C. Ransome, M. J. Darnley, S. M. Habergham-Mawson, M. W. Healy, P. A. James, S. C. Williams et al., “A Spectroscopic and Photometric Survey of Novae in M31 II.” 2020.

[82] K. Hornoch and A. W. Shafter, M31N 2006-11c appears to be spatially coincident with PNV J00413317+4110124 and hence a recurrent nova in M31, The Astronomer's Telegram 7116 (2015) .

[83] P. Schmeer, CBAT, Dec., 2017.

[84] S. C. Williams and M. J. Darnley, Spectroscopic classification AT 2017jdm as a nova, and likely recurrent eruption of M31N 2007-10b, The Astronomer's Telegram 11088 (2017) .

[85] P. Sin, M. Henze, G. Sala, A. Ederoclite, M. Hernanz, J. Jose et al., Additional Photometry for nova M31N 2016-12e and classification as a recurrent nova (= M31N 2007-11f), ATel, No. 100011 (2017) .

[86] A. W. Shafter, M. Henze, M. J. Darnley, R. Ciardullo, B. D. Davis and S. L. Hawley, The Recurrent Nova Candidate M31N 1966-08a = 1968-10c is a Galactic Flare Star, Research Notes of the American Astronomical Society 1 (2017) 44 [1712.05023].

[87] A. W. Shafter, M. J. Darnley, M. F. Bode and R. Ciardullo, On the Spectroscopic Classes of Novae in M33, ApJ 752 (2012) 156 [1204.4850].

[88] P. Mróz, A. Udalski, R. Poleski, I. Soszyński, M. K. Szymański, G. Pietrzyński et al., OGLE Atlas of Classical Novae. II. Magellanic Clouds, ApJS 222 (2016) 9 [1511.06355].

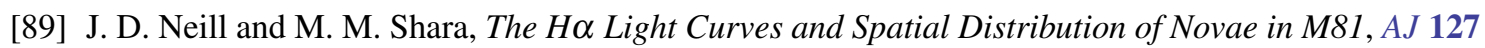
(2004) 816 [astro-ph/0311327]. 
[90] M. F. Bode, M. J. Darnley, A. W. Shafter, K. L. Page, O. Smirnova, G. C. Anupama et al., Optical and X-ray Observations of M31N 2007-12b: An Extragalactic Recurrent Nova with a Detected Progenitor?, ApJ 705 (2009) 1056 [0902.0301].

[91] M. J. Darnley, M. F. Bode, D. J. Harman, R. A. Hounsell, U. Munari, V. A. R. M. Ribeiro et al., On the Galactic Nova Progenitor Population, in Stellar Novae: Past and Future Decades, P. A. Woudt and V. A. R. M. Ribeiro, eds., vol. 490 of Astronomical Society of the Pacific Conference Series, (San Francisco), pp. 49-55, Dec., 2014, 1303.2711.

[92] N. P. M. Kuin, K. L. Page, P. Mróz, M. J. Darnley, S. N. Shore, J. P. Osborne et al., The January 2016 eruption of recurrent nova LMC 1968, MNRAS (2019) 2562 [1909. 03281 ].

[93] B. Warner, Cataclysmic variable stars. Cambridge Astrophysics Series, Cambridge, New York: Cambridge University Press, 1995, 1995.

[94] I. A. Steele, R. J. Smith, P. C. Rees, I. P. Baker, S. D. Bates, M. F. Bode et al., The Liverpool Telescope: performance and first results, in Ground-based Telescopes, J. M. Oschmann, Jr., ed., vol. 5489 of Society of Photo-Optical Instrumentation Engineers (SPIE) Conference Series, pp. 679-692, Oct., 2004, DOI.

[95] N. Gehrels, G. Chincarini, P. Giommi, K. O. Mason, J. A. Nousek, A. A. Wells et al., The Swift Gamma-Ray Burst Mission, ApJ 611 (2004) 1005.

[96] K. Page, A. Beardmore and J. Osborne, Neil gehrels swift observatory studies of supersoft novae, Advances in Space Research (2019).

[97] A. Merloni, P. Predehl, W. Becker, H. Böhringer, T. Boller, H. Brunner et al., eROSITA Science Book: Mapping the Structure of the Energetic Universe, arXiv e-prints (2012) arXiv:1209.3114 [1209.3114].

[98] Ž. Ivezić, S. M. Kahn, J. A. Tyson, B. Abel, E. Acosta, R. Allsman et al., LSST: From Science Drivers to Reference Design and Anticipated Data Products, ApJ 873 (2019) 111 [0805.2366].

[99] C. M. Copperwheat, I. A. Steele, R. M. Barnsley, S. D. Bates, D. Bersier, M. F. Bode et al., Liverpool telescope 2: a new robotic facility for rapid transient follow-up, Experimental Astronomy 39 (2015) 119 [1410.1731].

[100] R. D. Moore, D. Eick, H. Frand, R. E. French and M. Rymer, Battlestar Galactica: Season 4 - No Exit. NBCUniversal Television Distribution, 2009.

[101] K. Nishiyama and F. Kabashima, CBAT, Dec., 2008.

[102] A. Oksanen, M. J. Darnley, A. W. Shafter, S. Kafka, M. Kato and M. Henze, Recurrent Nova M31N 2008-12a: discovery of the 2019 eruption, The Astronomer's Telegram 13269 (2019) 1.

[103] M. Henze, J.-U. Ness, M. J. Darnley, M. F. Bode, S. C. Williams, A. W. Shafter et al., A remarkable recurrent nova in M 31: The X-ray observations, A\&A 563 (2014) L8 [1401.2904].

[104] S. Tang, L. Bildsten, W. M. Wolf, K. L. Li, A. K. H. Kong, Y. Cao et al., An Accreting White Dwarf near the Chandrasekhar Limit in the Andromeda Galaxy, ApJ 786 (2014) 61 [1401.2426].

[105] M. J. Darnley, R. Hounsell, P. Godon, D. A. Perley, M. Henze, N. P. M. Kuin et al., Inflows, Outflows, and a Giant Donor in the Remarkable Recurrent Nova M31N 2008-12a?-Hubble Space Telescope Photometry of the 2015 Eruption, ApJ 849 (2017) 96 [1 709.10145$].$

[106] R. E. Williams, E. P. Ney, W. M. Sparks, S. G. Starrfield, S. Wyckoff and J. W. Truran, Ultraviolet spectral evolution and heavy element abundances in nova Coronae Austrinae 1981., MNRAS 212 (1985) 753. 
[107] S. Starrfield, W. M. Sparks and J. W. Truran, Hydrodynamic Models for Novae with Ejecta Rich in Oxygen, Neon, and Magnesium, ApJ 303 (1986) L5.

[108] M. J. Darnley, R. Hounsell, P. Godon, D. A. Perley, M. Henze, N. P. M. Kuin et al., No Neon, but Jets in the Remarkable Recurrent Nova M31N 2008-12a?-Hubble Space Telescope Spectroscopy of the 2015 Eruption, ApJ 847 (2017) 35 [1708.06795].

[109] M. J. Darnley, M. Henze, M. F. Bode, I. Hachisu, M. Hernanz, K. Hornoch et al., M31N 2008-12a The Remarkable Recurrent Nova in M31: Panchromatic Observations of the 2015 Eruption., ApJ 833 (2016) 149 [1607.08082].

[110] M. J. Darnley, M31N 2008-12a - The Remarkable Recurrent Nova in M31, in 20th European White Dwarf Workshop, P.-E. Tremblay, B. Gaensicke and T. Marsh, eds., vol. 509 of Astronomical Society of the Pacific Conference Series, (San Francisco), pp. 515-520, Mar., 2017, 1611.01301.

[111] M. J. Darnley, M. Henze, A. W. Shafter, S. C. Williams, D. Boyd and K. Hornoch, "Back on Track? — The 2017 and 2018 eruptions of M31N 2008-12a.” 2020.

[112] M. J. Darnley, M. Henze, I. A. Steele, M. F. Bode, V. A. R. M. Ribeiro, P. Rodríguez-Gil et al., A remarkable recurrent nova in M31: Discovery and optical/UV observations of the predicted 2014 eruption, A\&A 580 (2015) A45 [1506.04202].

[113] M. J. Darnley, R. Hounsell, T. J. O’Brien, M. Henze, P. Rodríguez-Gil, A. W. Shafter et al., A recurrent nova super-remnant in the Andromeda galaxy, Nature 565 (2019) 460 [1712. 04872 ]. 